\title{
Successful resection of large mediastinal mature cystic teratoma immediately after evacuation of fluid content
}

This article was published in the following Dove Press journal:

Open Access Surgery

16 November 2010

Number of times this article has been viewed

\section{Ryo Miyahara \\ Shinjiro Nagai \\ Toshihiko Sato \\ Chen Fengshi \\ Toru Bando \\ Kenichi Okubo \\ Hiroshi Date}

Department of Thoracic Surgery, Kyoto University, Kyoto, Japan
Correspondence: Ryo Miyahara Department of Thoracic Surgery Kyoto University, 54 Kawahara-cho Shogoin

Sakyo-ku, Kyoto, Japan

Tel +8 I 7575 I 4975

Fax +8I 75 75। 4974

Email rmiyahar@kuhp.kyoto-u.ac.jp
Abstract: In this report, we presented a case of urgent resection of mature giant mediastinal teratoma. Its characteristic radiologic findings allowed us to plan evacuation of intratumoral fluid in order to make the surgical procedure safe and less invasive. In addition, a hybrid thoracoscopic-open approach also provided safety and allowed a somewhat less invasive tumor resection than otherwise would have been required. The patient recovered well without recurrence at nine months' follow-up, with full expansion of the right middle and lower lobes. Here we discuss the diagnosis and surgical procedure with reference to the literature.

Keywords: mediastinal mature teratoma, rupture, acute pleuritis

\section{Introduction}

Mature mediastinal teratoma (MMT) is the most common primary germ cell tumor in the mediastinum. ${ }^{1}$ Although patients with unruptured teratomas are often asymptomatic, up to $36 \%$ of all MMTs rupture and cause severe symptoms, depending on tumor size and site of rupture. ${ }^{2,3}$ Urgent resection with extensive median sternotomy and/or thoracotomy is mandatory in the case of massive tumors. ${ }^{4}$ We report a case of a huge MMT with distinctive radiographic features that was successfully managed through sequential steps, allowing an overall approach of limited invasiveness.

\section{Case report}

A 32-year-old woman was admitted to our outpatient clinic because of back discomfort for one week after undertaking vigorous exercise. A chest radiograph showed a huge mass in the right pleural cavity with a fat-fluid level (Figure 1a). Computed tomography demonstrated a massive cystic lesion with a fat-fluid level interface containing soft tissue-density nodules. This cystic lesion occupied nearly the entire right pleural cavity, and its mass effect produced a severe mediastinal shift. Together, all these findings were consistent with a huge MMT growing rightward from the anterior mediastinum (Figure 1b, 1c, and 1d). We planned for resection of the tumor to be done the following week. However, one day later she presented as an emergency with significantly worsened back pain accompanied by orthopnea. A chest radiograph at this time showed a decrease of aerated right lung with a fluid shift suggesting spontaneous laceration of the tumor cyst wall (Figure 1e). Her respiratory rate and heart rate were 32 per minute and 99 per minute, respectively. Abnormal laboratory data included a white blood cell count of $12,800 / \mathrm{mm}^{3}$ with $77 \%$ polymorphonuclear leukocytes, elevated C-reactive protein at $23.8 \mathrm{mg} / \mathrm{dL}$, and a markedly elevated serum CA19-9 value at 12,857.5 U/mL. Together 

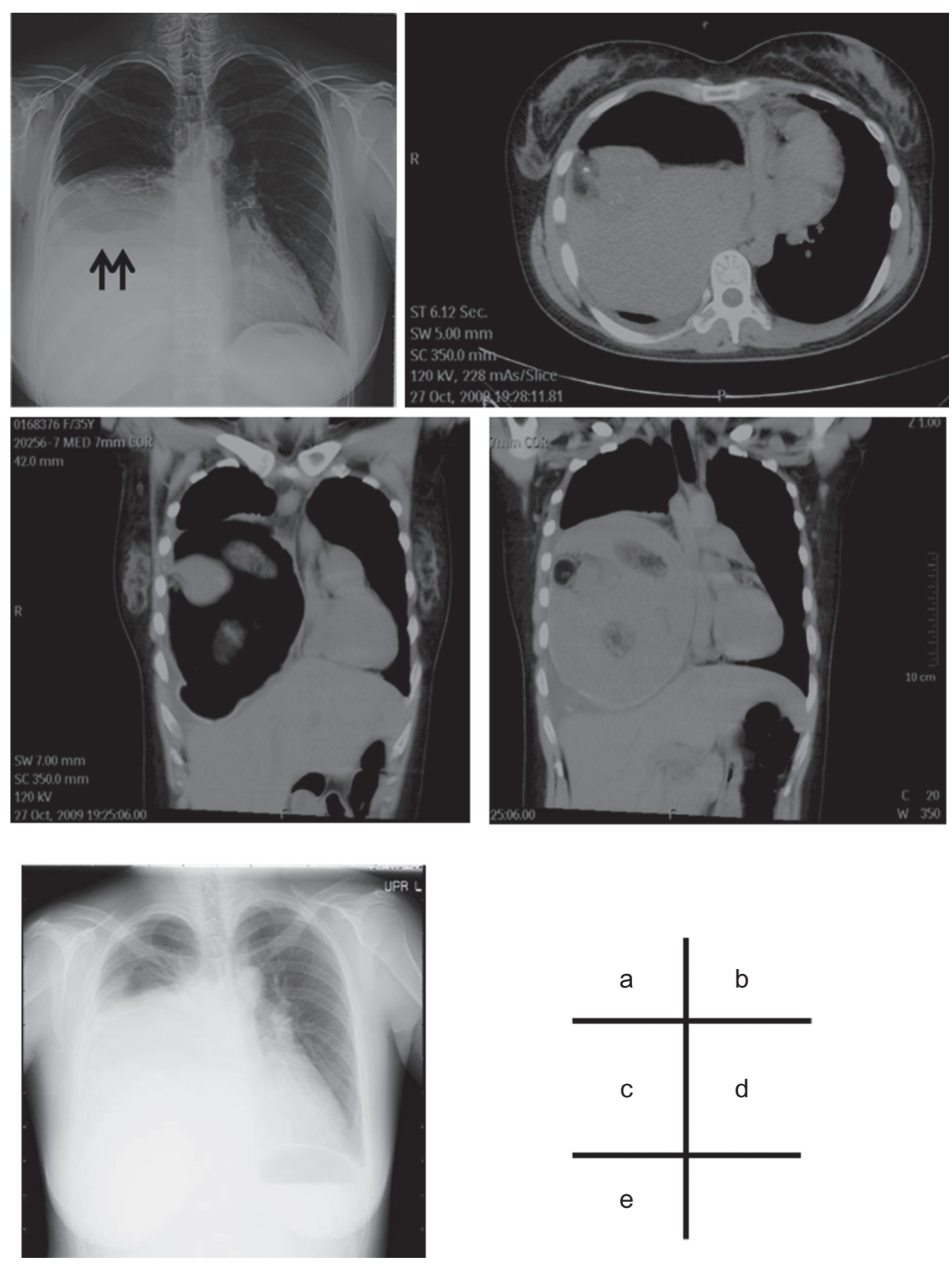

Figure I a) Chest radiograph at initial admission; fluid level can be observed. b) Axial view of CT at ninth vertebral level; oil-fluid level can also be observed. c) and d) show coronal views; border surface of two different fluids can be observed throughout almost all of the tumor, suggesting that the cystic cavity contained two different fluids, with some nodular components floating at their interface. e) Chest radiograph at emergency admission; decreasing right lung area and increasing mediastinal shift to left side suggested rapid collection of pleural effusion.

with blood gas analysis showing relative hypoxia $\left(\mathrm{PaO}_{2} 61\right.$ torr $)$ and severe inflammation, the patient's condition was consistent with systemic inflammatory response syndrome (SIRS), prompting emergency tumor resection. It seemed dangerous to place the patient in the left decubitus position initially because of the anticipated mass effect of the tumor, and it was hoped that preliminary fluid evacuation would render tumor resection much safer by diminishing left lung compression during left lateral positioning. Therefore, we first performed a right anterior minithoracotomy with the patient lying on her back but positioned as upright as possible (Figure 2a). Approximately $2700 \mathrm{~mL}$ of fatty fluid was evacuated (Figure 2b). Computed tomography scan suggested that there were two distinct fluid collections, one comprising transparent fatty liquid and the other made up of opaque fluid. We then turned the patient to the left lateral decubitus position, allowing a limited right 

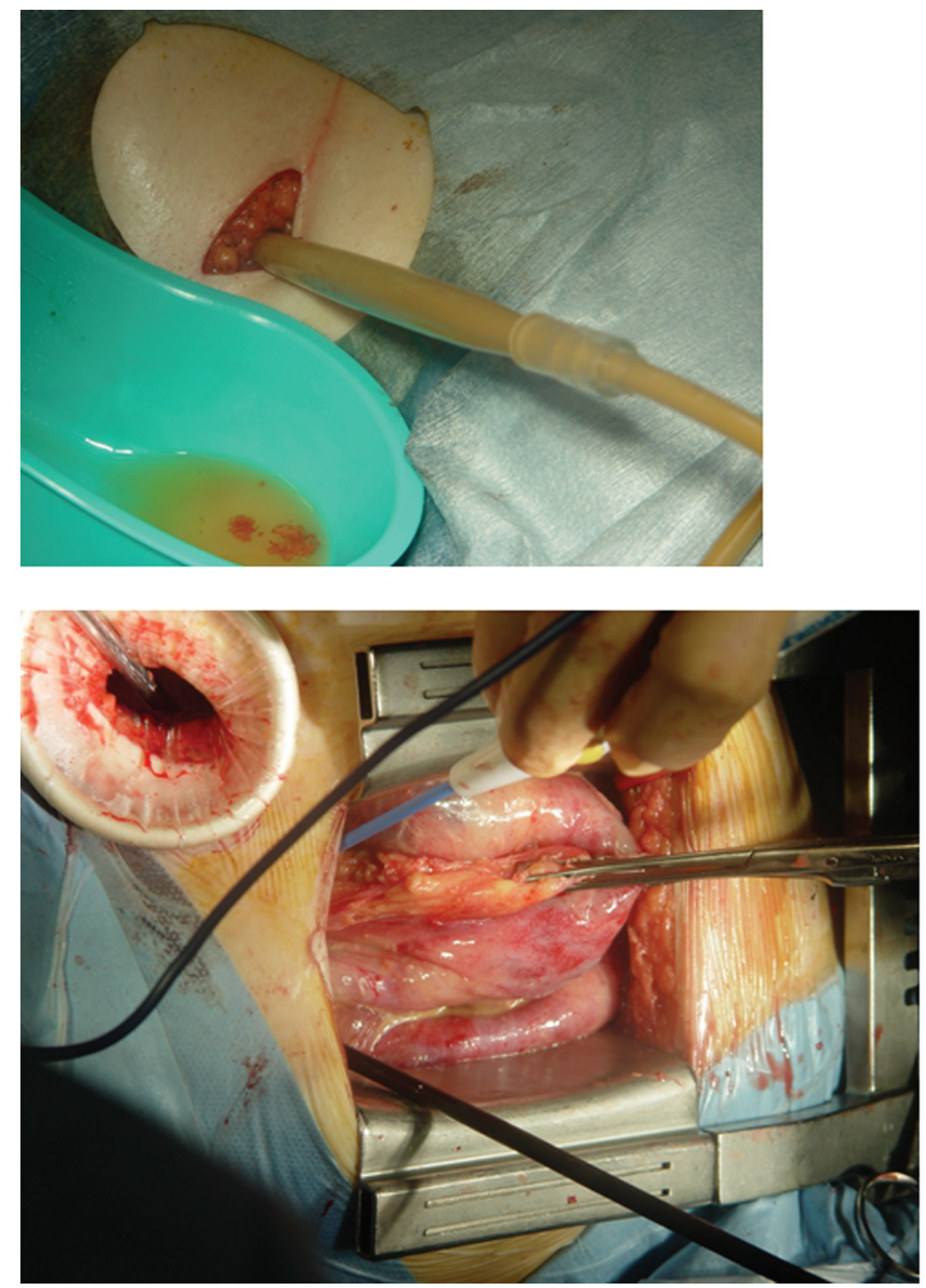
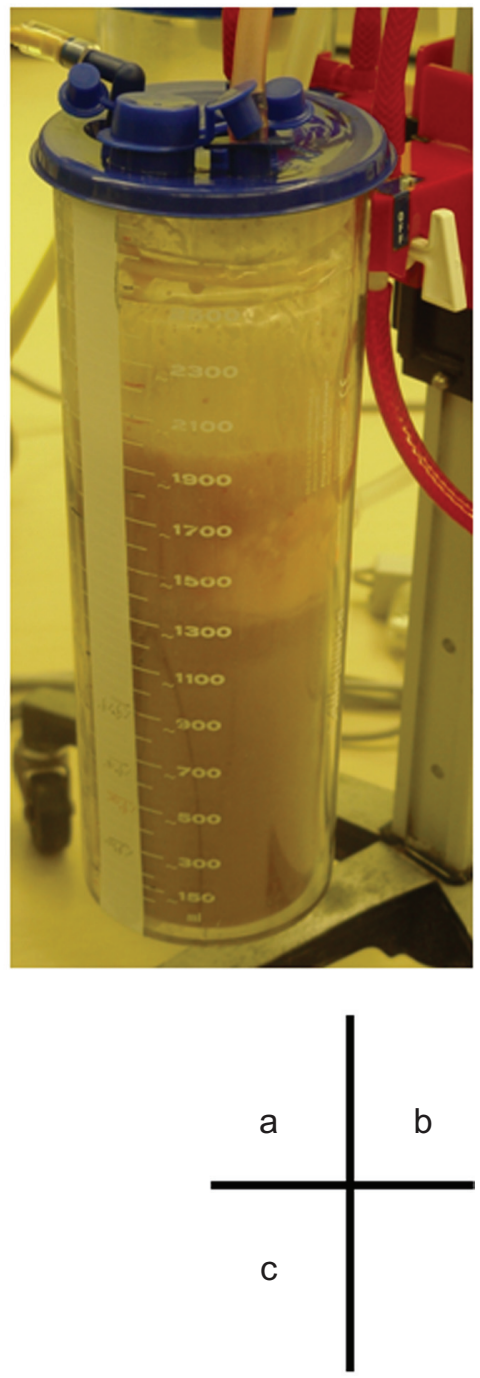

Figure 2 a) and b) Minithoracotomy was done to decompress the tumor by aspiration of $2 \mathrm{~L}$ of thick opaque fatty fluid and $0.7 \mathrm{~L}$ of transparent fatty fluid. c) Evacuation of intratumoral fluid enabled resection of thick fibrous tumor wall from mediastinum under assistance of view of video assisted thoracoscopy.

posterolateral thoracotomy. Through this we safely resected a thick fibrous capsule along with the right lobe of thymus via a sixth interspace thoracotomy using video thoracoscopic assistance (Figure 2c). By placing a videoscope through the initial anterior minithoracotomy site, we were able to visualize the upper mediastinal aspect of the tumor clearly in a way that we could not through the limited posterolateral thoracotomy. In particular, the right phrenic nerve was readily identified and preserved during the cephalocaudal tumor resection. This hybrid thoracoscopic-open approach provided safety and allowed a somewhat less invasive tumor resection than otherwise would have been required.

The resected tumor measured $18.5 \times 16 \times 5 \mathrm{~cm}$ and consisted of thick fibrous tissue surrounding contents of hair, fat, bone, and skin (Figure 3a). Histologically, the lesion contained skin components, with sebaceous glands, vessels, fat, and muscle with cartilage and bone (Figure $3 b$ and $3 c$ ). The chest tube was removed on postoperative day 5 , and the patient was discharged home on postoperative day 9 without complications. She recovered well without recurrence at nine months' follow-up, with full expansion of the right middle and lower lobes, and a normalized CA19-9 level at $44.3 \mathrm{U} / \mathrm{mL}$.

\section{Discussion}

Mature mediastinal teratoma is an uncommon primary germ cell neoplasm composed of more than one of the three primitive germ cell layers. Although patients with unruptured teratomas are often asymptomatic, up to $36 \%$ of 

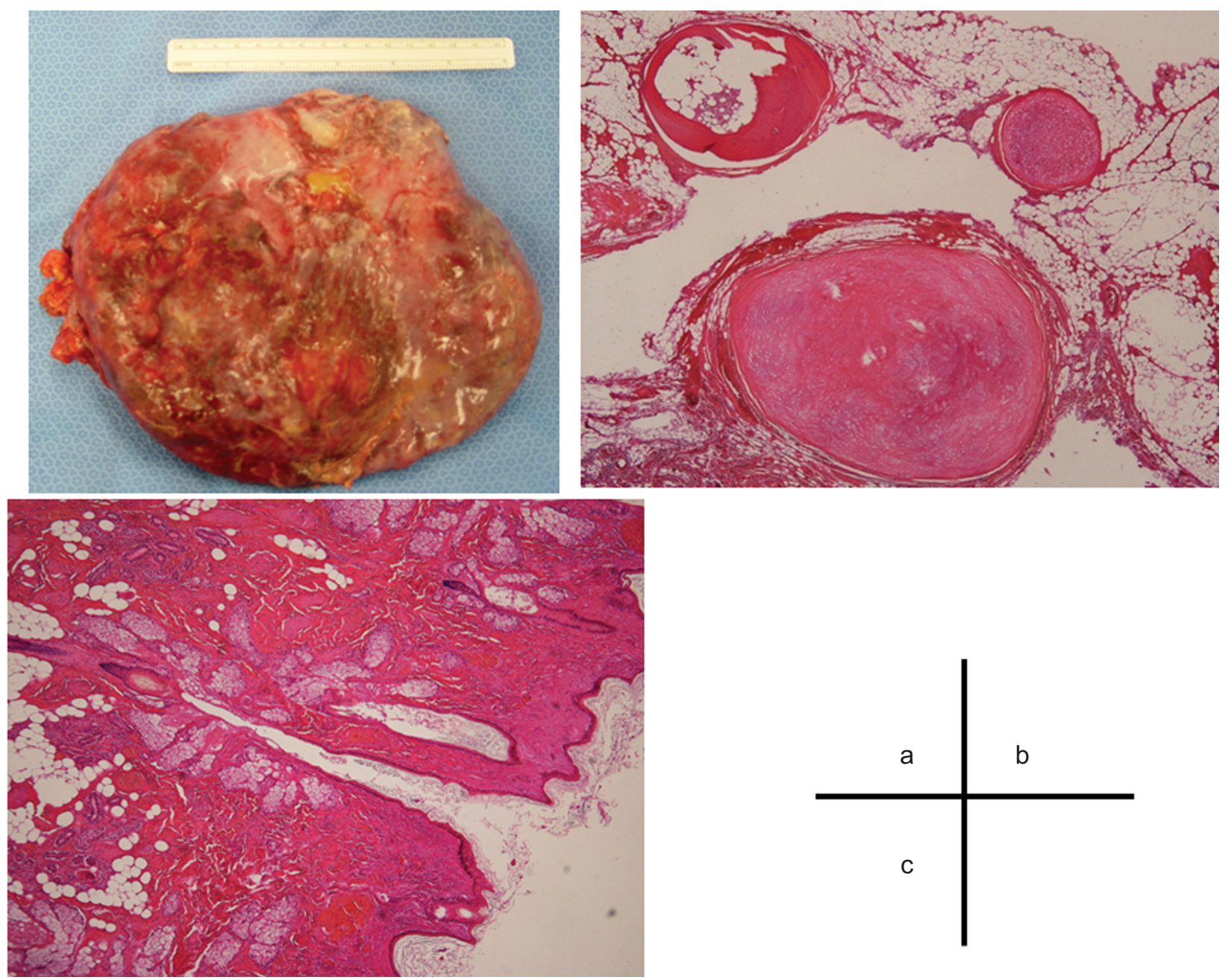

Figure 3 a) Excised specimen. b) and c) Histologically, the specimen contained skin components with sebaceous glands, vessels, and fat, and bone and cartilage.

all reported MMTs rupture and cause severe symptoms, such as chest and/or back pain, fever, cough productive of hairy and/or sebaceous expectorant, and even cardiac tamponade. The patient in our case eventually presented with SIRS likely secondary to leakage of sebaceous fluid into the pleural space. Several hypotheses have been proposed regarding the cause of spontaneous rupture into the pleural cavity, including the action of digestive enzymes secreted from intratumoral glands, ischemia, pressure necrosis, and infection. ${ }^{3}$ Because our patient's symptoms developed following vigorous exercise, mechanical cyst laceration with sebaceous fluid spillage was the most probable event. Because both fluids congealed at room temperature within one hour of drainage, we could not analyze the components fully. Although fluid cultures were not done, the odorless and lipid-like character of the fluid suggested chemical irritation rather than infection as the cause of the SIRS.
On radiologic imaging, MMTs are generally described as masses of heterogeneous attenuation with varying combinations of soft tissue, fluid, fat, and calcium. ${ }^{5}$ Interestingly, in our case, radiographic findings indicated that the tumor had a large single cavity filled with two kinds of fluid, and we were able to evacuate most of the fluid safely just prior to definitive tumor resection. This also allowed performance of less morbid incisions than otherwise may have been required. There are reports of MMTs showing malignant transformation that is further complicated by spontaneous rupture, or inadvertent rupture during surgery, associated with early pleural relapse of malignant components. ${ }^{6}$ Although these events are extremely rare, it would seem prudent to proceed to careful MMT resection as soon as practical after diagnosis to prevent pleural seeding of malignant elements in the event of spontaneous tumor rupture. 


\section{Acknowledgment}

We deeply appreciate the writing assistance of Dr Van Natta for the English version of this manuscript.

\section{Disclosure}

The authors report no conflicts of interest in this work.

\section{References}

1. Priola AM, Priola SM, Cardinale L, Cataldi A, Fava C. The anterior mediastinum: Diseases. Radiol Med. 2006;111:312-342.

2. Rosado-de-Christenson ML, Templeton PA, Moran CA. Mediastinal germ cell tumors: Radiologic and pathologic correlation. Radiographics. 1992;12:1013-1030.
3. Sasaka K, Kurihara Y, Najakima Y, et al. Spontaneous rupture: A complication of benign mature teratomas of the mediastinum. Am J Roentgenol. 1998;170:323-328.

4. Shikata H, Matsubara J, Tsuchishima S, et al. The case of a mediastinal huge teratoma in a 12-year-old girl with the extremely increased CA19-9 level [in Japanese]. J Jap Association Chest Surg. 2000;14:649-654.

5. Moeller KH, Rosado-de-Christenson ML, Templeton PA. Mediastinal mature teratoma: Imaging features. Am J Roentgenol. 1997;169:985-990.

6. Chang CC, Chang YL, Lee YC. Cystic malignant teratoma with early recurrence after intraoperative spillage. Ann Thorac Surg. 2008;86:1971-1973.
Open Access Surgery

\section{Publish your work in this journal}

Open Access Surgery is an international, peer-reviewed, open access journal that focuses on all aspects of surgical procedures and interventions. Patient care around the peri-operative period and patient outcomes post surgery are key topics. All grades of surgery from minor cosmetic interventions to major surgical procedures are covered. Novel techniques

\section{Dovepress}

and the utilization of new instruments and materials, including implants and prostheses that optimize outcomes constitute major areas of interest. The manuscript management system is completely online and includes a very quick and fair peer-review system. Visit http://www.dovepress.com/ testimonials.php to read real quotes from published authors. 